\title{
Mejora en el tiempo de atención al paciente en una unidad de urgencias gineco-obstétricas mediante la aplicación de Lean Manufacturing*
}

\author{
Paloma Martínez Sánchez**, José Martínez Flores***, \\ Pablo Nuño De La Parra ****, Judith Cavazos Arroyo*****
}

\begin{abstract}
Resumen
Introducción. Se presenta una propuesta de mejora en el tiempo de atención al paciente, mediante la aplicación de la metodología de Lean Manufacturing, en una unidad de urgencias de gineco-obstetricia en una clínica en Bogotá, la cual presenta exceso en los tiempos de espera por parte de los pacientes frente a los estándares establecidos en la Ley 237 de 2008 del Senado de la República. Objetivo. Desarrollar una propuesta de mejora en los tiempos de atención por medio de la metodología Lean Manufacturing en la unidad de urgencias gineco-obstétricas de la clínica $A B C$. Materiales y métodos. Se realizó un trabajo de campo durante 6 meses, en los cuales mediante el uso de herramientas propias del lean manufacturing, tales como el value stream mapping y el diagrama de spaghetti, así como con un estudio de tiempos a una muestra representativa de los pacientes, se identificaron las áreas y/o procesos que no agregan valor al paciente generando planes de acción que contribuyeran a la mejora en los tiempos de atención. Resultados. La aplicabilidad de la propuesta se evaluó a través de simulaciones basadas en el software Arena, demostrando mejoras en los tiempos de atención de hasta el 56 \%. Conclusión. Este proyecto evidencia la aplicabilidad del Lean manufacturing y sus principales herramientas, en clínicas u hospitales en Bogotá, permitiendo establecer los procesos que necesitan ser mejorados o eliminados con el fin de disminuir los tiempos de atención al paciente.
\end{abstract}

Palabras clave: lean manufacturing, unidad de urgencias, tiempos de atención, simulación.

\section{Improvement in the time of attention to patients in a gyneco-obstetrics emergency unit by means of the application of lean manufacturing}

\begin{abstract}
Introduction. This paper introduces a proposal to improve the time of attention for patients, by means of the Lean Manufacturing methodology, in a gyneco-obstetrics emergency unit located in Bogotá, in which the patients have to wait for a long time and this fact goes against law 237, 2008, produced by the Colombian Senate. Objective. Develop an improvement proposal for the attention time, by the use of the Lean Manufacturing methodology, in the gyneco-obstetrics emergency unit of the ABC Clinic. Materials and methods. A field work was developed during six months, in which, by the use of Lean Manufacturing tools such as the value stream mapping and the spaghetti diagram and a study of times on a representative sample of patients, the areas and processes that do not add value to those patients were identified and, then, action plans to improve the attention times were generated. Results. The applicability of the proposal was assessed by means of simulations based on the Arena software, demonstrating improvements
\end{abstract}

\footnotetext{
* $\quad$ El artículo se enmarca dentro de una investigación de estudio casos relacionada con el proyecto doctoral propuesto por Paloma Martínez Sánchez y financiado por la Universidad El Bosque.

** PhD en Logística y Cadena de Suministro. Máster en Ciencias con especialidad en Sistemas de Calidad y Productividad. Docente e investigadora, grupo de investigación GINTECPRO, Universidad El Bosque, Bogotá, Colombia.

*** PhD en Ingeniería

$\star * * * \quad$ PhD en Ingeniería Industrial

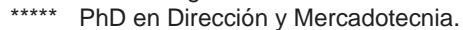

Autor para correspondencia: Paloma Martínez Sánchez, e-mail: martinezpaloma@unbosque.edu.co Artículo Recibido:10/07/2015 Artículo Aprobado: 15/11/2016 
in the attention times of up to $56 \%$. Conclusion. This project demonstrates the applicability of Lean Manufacturing and its main tools in clinics or hospital in Bogotá, contributing to establish what processes must be improved or eliminated in order to reduce the attention time for the patients.

Key words: lean manufacturing, emergency unit, attention time, simulation.

\section{Melhora no tempo de atendimento ao paciente em uma unidade de urgências gineco-obstétricas mediante a aplicação de lean manufacturing}

\section{Resumo}

Introdução. Se apresenta uma proposta de melhora no tempo de atendimento ao paciente, mediante a aplicação da metodologia de Lean Manufacturing, em uma unidade de urgências de gineco-obstetrícia em uma clínica em Bogotá, a qual apresenta excesso nos tempos de espera por parte dos pacientes frente aos padrões estabelecidos na Lei 237 de 2008 do Senado da República. Objetivo.
Desenvolver uma proposta de melhora nos tempos de atendimento por meio da metodologia Lean Manufacturing na Unidade de Urgências ginecoobstétricas da Clínica ABC. Materiais e métodos. Se realizou um trabalho de campo durante 6 meses, nos quais mediante o uso de ferramentas próprias do lean manufacturing, tais como o value stream mapping e o diagrama de spaghetti, assim como com um estudo de tempos a uma amostra representativa dos pacientes, se identificaram as áreas e/ou processos que não agregam valor ao paciente gerando planos de ação que contribuíram na melhora nos tempos de atendimento. Resultados. A aplicabilidade da proposta se avaliou através de simulações baseadas no software Arena, demostrando melhoras nos tempos de atenção de até $56 \%$. Conclusão. Este projeto evidencia a aplicabilidade do Lean manufacturing e suas principais ferramentas, em clínicas ou hospitais em Bogotá, permitindo estabelecer os processos que necessitam ser melhorados ou eliminados com o fim de diminuir os tempos de atendimento ao paciente.

Palavras chave: lean manufacturing, unidade de urgências, tempos de atendimento, simulação.

\section{Introducción}

El lean es reconocido como una metodología de mejoramiento continuo (Manos et al., 2006; Gifford, 2008; Zarbo, 2012; Culcuoglu, 2011) enfocada en la reducción de los desperdicios o muda (Zarbo, 2012). Los muda son clasificados como: espera, defectos, movimientos innecesarios, exceso de inventario, sobreproducción, exceso de transporte y sobre-procesamiento (Weinstock, 2008; Cachon \& Terwiesch, 2009; Waring \& Bishop, 2010).

Esta metodología se considera aplicable a los procesos relacionados con la salud (Kollberg et al., 2007; Brandao, 2009; Papadopoulos et al, 2011., La Ganga, 2011; Mazzocato et al., 2012), así como con los departamentos de emergencia (Piggott et al., 2011; Mazzocato et al., 2012; Martínez et al., 2014; Martínez et al., 2015) debido a que mediante esta los procesos son llevados a cabo con calidad, y cumpliendo con la satisfacción del consumidor (Weinstock, 2008; Morales, 2010; Mannon, 2014). Los desperdicios o muda en los servicios de salud son vistos como actividades que no añaden valor al paciente (Pondhe et al., 2006; Bendavid et al., 2012; Reijula y Tommelein, 2012), tal como es el caso de los largos tiempos de espera para ser asistidos (Anneke y Dadich, 2009).

En países desarrollados (Adra, 2005; Aherne, 2007; Bliss, 2009; Brandao, 2009; Sobek y Lang., 2010; Burgess y Radnor, 2013; Breuer, 2013), así como en aquellos considerados como emergentes, se ha comenzado a aplicar lean en servicios de salud (Varkey y Kollengode, 2011; Ponanake et al., 2014; Pestana et al., 2014), pero en países como Colombia estas metodologías son recientes, presentando su aplicación principalmente en empresas de manufactura (Dinas et al., 2009; Arrieta et al., 2010).

A pesar de que la salud es un bien y un derecho fundamental de las personas (Herazo, 2010), en ocasiones se ve socavada por la prestación de un servicio no adecuado por las 
entidades prestadoras de salud (IPS), lo cual se traduce en fallas y quejas de los usuarios (Argas et al., 2010). En el ámbito internacional, se establece que el departamento o unidad de emergencias es el más ineficiente (Dickson et al., 2009), por lo que requiere mejoras (Holden, 2011). Colombia no es ajena a dicha situación, tal como establecen Lopera et al. (2010) en un estudio realizado en la ciudad de Medellín, en el cual se determina que existe una serie de barreras en esta unidad que no permite que los pacientes reciban una adecuada atención.

Este estudio hace parte de una investigación doctoral, basada en estudios de casos, realizada en Unidades de Urgencia en clínicas u hospitales en Bogotá y La Sabana de Bogotá, cuyo objetivo era determinar si el lean podía ser considerado como una metodología válida de aplicación en el contexto colombiano, así como establecer los principales aspectos que generan demora en la atención, proporcionando mejoras en los procesos con miras a fortalecer la percepción de valor para los usuarios (Wood, 2004; Lewis, 2012; Found y Harrison, 2012).

\section{Materiales y métodos}

Se desarrolla una investigación en campo para establecer el estado actual de los procesos en la unidad de urgencias estudiada y así poder diseñar una propuesta de mejora, con el fin de disminuir los tiempos de atención a los pacientes, para lo cual, en primera instancia, se recolecta información por medio de visitas a la clínica, entrevistas a líderes y observación en sí del proceso, tomando los tiempos y recorridos realizados por 115 pacientes. Posteriormente, se analiza la información recolectada y se identifican las actividades que generan desperdicios y que no agregan valor (mudas), las cuales fueron representadas mediante un value stream mapping, con sus respectivos estallidos Kanban. Finalmente, se propone la mejora en los procesos centrados en los mudas y se valida la solución mediante el simulador Arena.

\section{Resultados}

Se realizó una entrevista estructurada con el jefe del departamento con el fin de establecer el proceso de atención a los pacientes, tal como se muestra en la figura 1 , en la cual se observan 8 pasos generales que deben seguir los pacientes para ser atendidos en la unidad de urgencias, desde su ingreso hasta la orden de salida. Es importante aclarar que las mujeres que asisten en estado de embarazo requieren atención inmediata y prioritaria; por lo tanto, no deben cumplir los pasos 3, 4 y 5, omitiendo la valoración en triage y el suministro de información sobre la paciente ante la ventanilla de admisiones (suministro que puede otorgar el acompañante).

De igual forma, se desarrolló un diagrama de flujo del área de urgencias gineco-obstétricas (figura 2) con el fin evidenciar el proceso que deben seguir las pacientes que ingresan a esta unidad y el flujo de información.

Posteriormente, se recolectaron los datos de las pacientes que acudieron a urgencias en la unidad de gineco-obstetricia durante los últimos tres años; se encontró que el mayor ingreso fue de 1020 pacientes; con esta cifra se calculó el tamaño de la muestra y se generó un valor de 64 tomas de tiempos; sin embargo, con el fin de tener una mayor fiabilidad del estudio, se hicieron 115 mediciones, debido a que la institución no contaba con información en relación con el número de pacientes por jornada, por lo cual se ejecutaron mediciones acordes con los turnos de trabajo: de 7 a. m. a 1 p. m; de 1 p. m. a 7 p. m. y de 7 p. m. a 7 a. $\mathrm{m}$. y se evidenció que en el primer turno se registraron 69 pacientes; en el segundo, 22, y en el tercero, 24.

En la figura 3, a continuación, se muestra el diagrama de Spaguetti en el cual se observan los recorridos realizados por las pacientes, donde se registran distancias de recorrido de hasta 92,74 metros.

De las 115 muestras, se tomaron únicamente aquellos datos de las pacientes que no fueron hospitalizadas debido a que este proceso es diferente al de urgencias gineco- obstétricas; allí se muestra que en promedio el tiempo de permanencia de la paciente era de 5 horas. Al realizar el estudio, se evidenciaron problemas relacionados con los siguientes aspectos: 

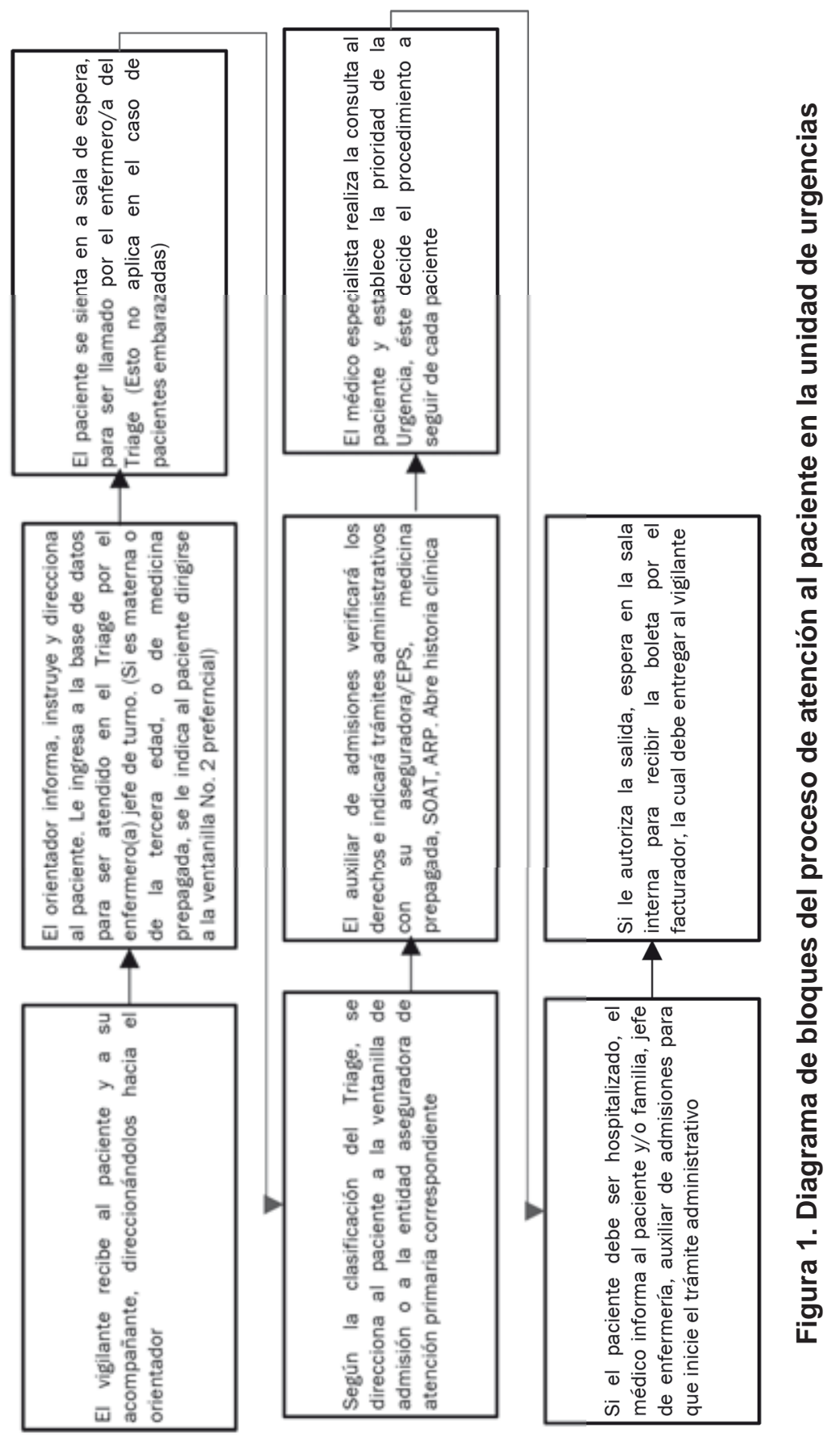

Mejora en el tiempo de atención al paciente en una unidad de urgencias gineco-obstétricas mediante la aplicación... 


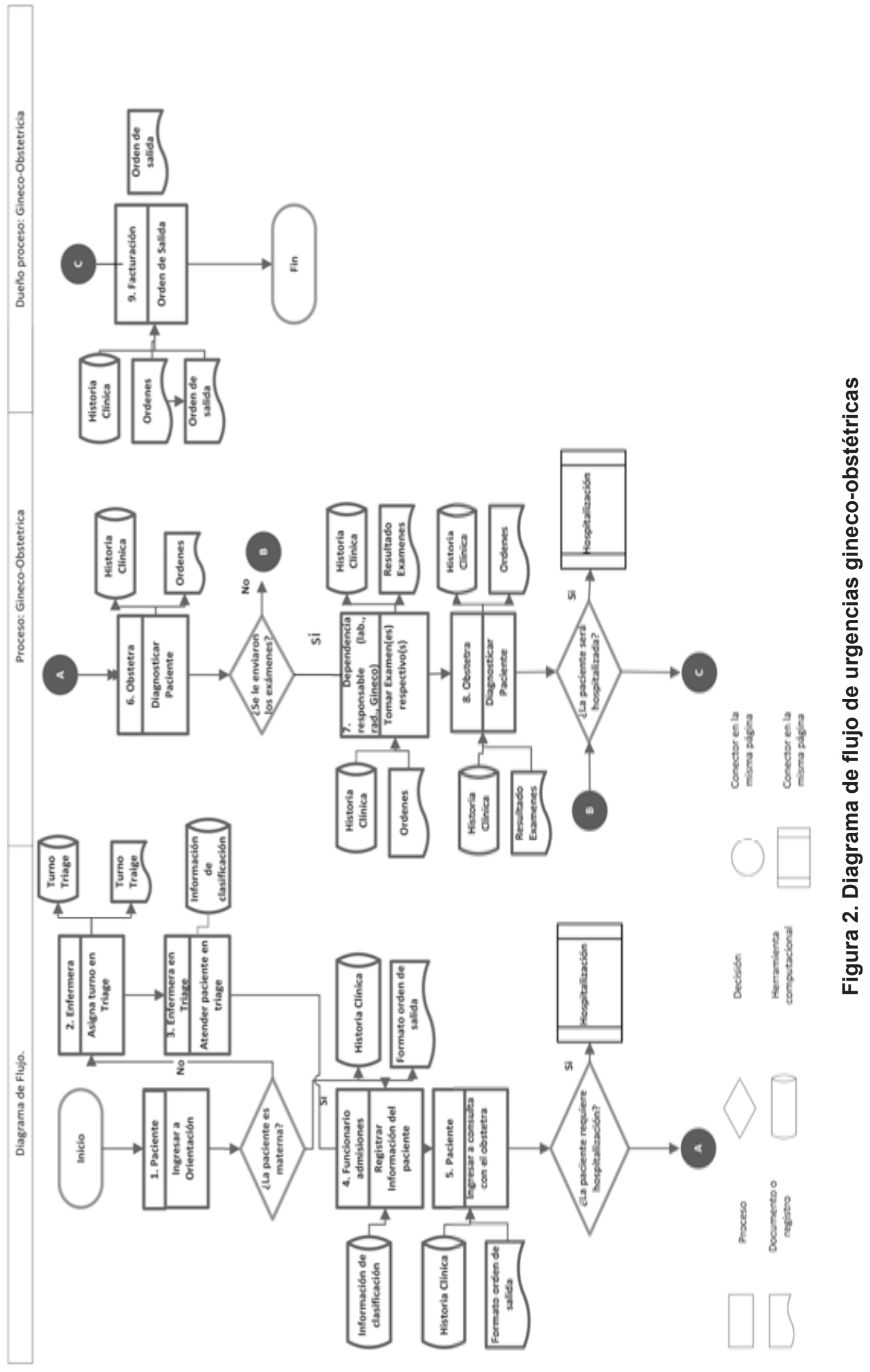




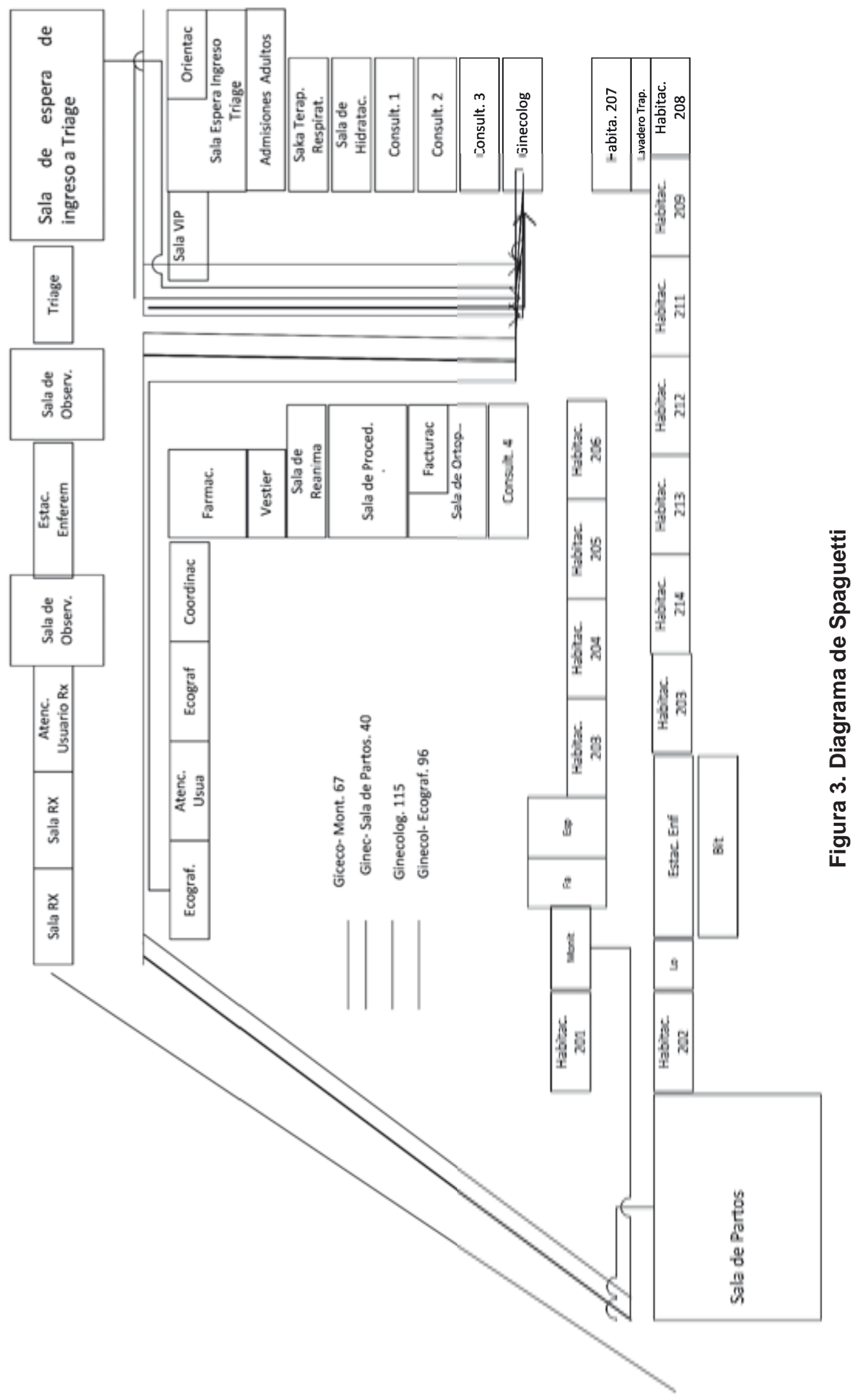


- Registro del paciente. En ocasiones, este no se encuentra registrado en el sistema, por lo tanto, no es llamado para realizar sus procedimientos.

- Información en el flujo de órdenes. Debido a que se maneja la orden de forma física, se generan pérdidas de tiempo por el transporte de estas a cada área, por ejemplo, la orden de facturación que el médico debe llevar a esta dependencia para que el paciente pueda egresar de la institución.

- Falta de personal. Se genera congestión y acumulamiento de órdenes en diferentes áreas, tal como es el caso de radiología, cuyo servicio es subcontratado por la entidad, y en el turno de 12 p. m. hasta las 7 a. m. no hay radiólogo, por ende, hay una acumulación de órdenes para el turno de la mañana.

En la figura 4, se observa el value stream mapping con los estallidos kanban, los cuales evidencian aspectos a mejorar.

Tomando en cuenta los estallidos Kanban, que se observan en la figura 4 basados en los tiempos y análisis de los diferentes procesos, se evidencia que los procesos a mejorar o eliminar, debido a que no agregan valor al servicio de urgencias gineco- obstétricas, son:

- Ingreso a consulta

- Procedimientos médicos

- Orden de salida

Se plantea la eliminación de la actividad de orientación, ya que este proceso es similar al que se hace en admisiones, con la diferencia de que en el primero se brinda el digiturno a la paciente, el cual puede ser tomado directamente por esta, suprimiendo la duplicidad en la captura de información. De igual forma, se evidencia que las personas de las diferentes áreas deberán ingresar al software Servintec (software de la clínica), los diferentes procesos, de tal forma que se pueda generar seguimiento a las pacientes en tiempo real y que estas puedan ser llamadas a las áreas pertinentes según las órdenes médicas en el sistema, tal como es el caso de entrega de orden a facturación por parte del médico, la cual ya se puede realizar directamente por el sistema.

Debido a la carencia de personal médico especializado (radiólogo) en los horarios de alta demanda, se observa que es necesaria la contratación de 1 radiólogo y 1 transcriptora adicional en el horario de 7 a. m. a 1 p. m., así como de 12 p. m. a 7 a. m., horarios en los cuales o no se cuenta con el apoyo de este personal, o se producen tiempos de espera por parte de las pacientes de hasta 5 horas; lo anterior, con la finalidad de prestar calidad y continuidad en el servicio.

De igual forma, se plantearon otras mejoras como es el caso de la redistribución de algunos espacios físicos, de manera que se permita un menor desplazamiento por parte del radiólogo para la entrega de la grabación a la transcriptora, así como la reubicación del área de monitorización con el fin de reducir los $92.74 \mathrm{~m}$ que deben recorrer las pacientes para este procedimiento.

Con el fin de determinar las mejoras propuestas en los tiempos de atención a las pacientes, se hizo una simulación en Arena en la cual se observan reducciones en el tiempo de permanencia de estas en la clínica de hasta un $56 \%$ del tiempo actual. 


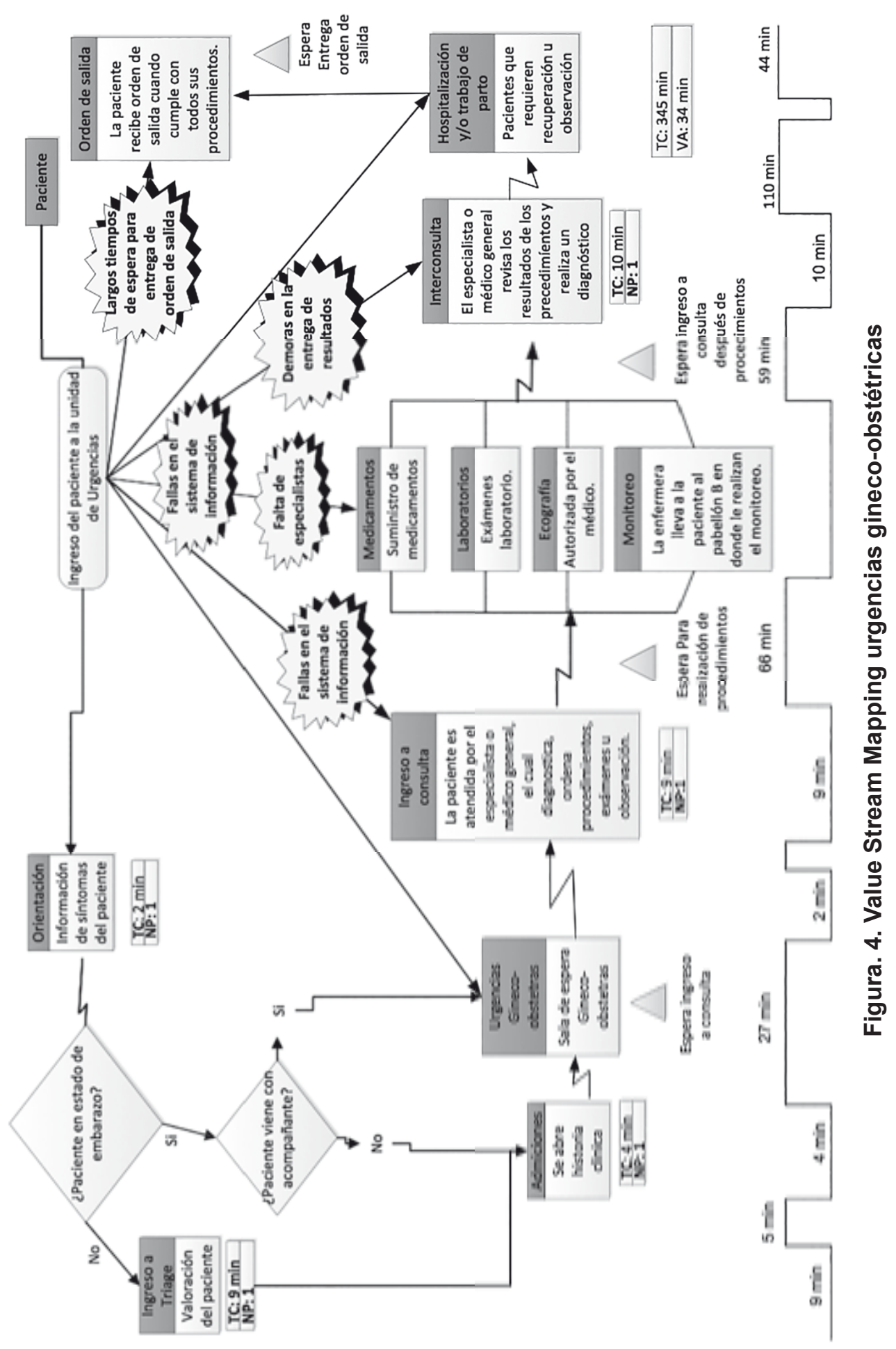

Mejora en el tiempo de atención al paciente en una unidad de urgencias gineco-obstétricas mediante la aplicación... 


\section{Discusión}

En la clínica analizada se identificó desconocimiento por parte del personal de las metodologías de mejora, tal como es el caso de Lean, así como del aprovechamiento de espacios para disminuir las distancias recorridas por pacientes. De igual forma, se observó que es vital realizar capacitaciones y re-inducciones al personal de clínica, estableciendo claramente las funciones a desempeñar, así como incentivarlos hacia el adecuado uso del software, con el fin de mejorar la agilidad en la prestación del servicio y emplear idóneamente las herramientas tecnológicas disponibles para tal fin.

Se demostró que, en el caso analizado, se pueden generar reducciones en los tiempos de espera y permanencia de las pacientes, lo cual permite reafirmar la meta principal de Lean hacía la generación de valor (Brandao, 2009; Lewis, 2012).

Por otra parte, es importante precisar que los resultados y conclusiones de este trabajo fueron validados por el personal médico del hospital, haciendo claro que estos no eran conscientes de algunas de sus debilidades.

\section{Conclusiones}

Mediante la aplicación de los tres pasos fundamentales del Lean establecidos por Dickson et al. (2009), se logró establecer las actividades o mudas que no generan valor al cliente (Solís y Madriz, 2009), eliminando los procesos que se encuentran duplicados (Radnor et al., 2011, Martinez et al., 2015). De igual forma, por medio de la redistribución en planta de la unidad de urgencias de gineco-obstetricia se logra reducir distancias de recorrido y tener un mejor flujo para las pacientes de la unidad.

El uso de herramientas de lean, tales como los mapas de valor y el diagrama de spaghetti, similar al caso planteado por Dammand et al., (2014) y Martínez et al. (2015), facilitaron la identificación de procesos y distancias a mejorar para la disminución en los tiempos de espera por parte de los pacientes.
A lo largo de esta investigación, se realizó la búsqueda de literarura relacionada con la aplicación de la manufactura esbelta en la asistencia sanitaria en países de América Latina, y se pudo confirmar lo expuesto por Moreno y Mendoza (2012), así como por Toussaint y Berry, (2013), quienes vislumbran la necesidad de incrementar los esfuerzos de investigación en esta área, ya que se pueden consideran como incipientes.

Para trabajos futuros en el área, se deberán proponer nuevos casos de estudio, que permitan evaluar diferencias en el impacto del Lean en estos, así como generar investigaciones en el área de inventarios, ya que se evidencia desconocimiento por parte del hospital en estos procesos al ser manejados, muchos de estos, por outsourcing.

\section{Agradecimientos}

Los autores agradecen la financiación de la Universidad El Bosque del proyecto de investigación "La manufactura esbelta en los departamentos de emergencias en IPS estudio de casos", siendo uno de los casos de estudio el aquí presentado. De igual forma agradecen a los estudiantes Natalia Velásquez y Edward Pérez, quienes mediante la ejecución de su trabajo de grado apoyaron el desarrollo del proyecto en mención.

\section{Referencias bibliográficas}

- Aherne, J. (2007). Think lean. Nursing Management, 13(10), 13-5.

- Adra, H. (2005). Applying lean to the healthcare sector (presentation). IIE Annual Conference. Proceedings, 1-21

- Anneke, J. y Dadich, A. (2009). Using Visual Analytics to Improve Hospital Scheduling and Patient Flow. Red de Revistas Científicas de América Latina, el Caribe, España y Portugal, 4(2), 20-30.

- Arlbjørn, J. y Freytag, P. (2013). Evidence of lean: A review of international peerreviewed journal articles. European Business Review, 25(2), 174-205.

- Arrieta, J.; Botero, V. y Romano, M. (2010). Benchmarking Sobre Manufactura Esbelta en el 
Sector de la Confección. Journal of Economics, Finance and Administrative Science, 15(28). 141-171.

- Bendavid, Y., Boeck, H., y Philippe, R. (2012). RFID-enabled traceability system for consignment and high value products: A case study in the healthcare sector. Journal of Medical Systems, 36(6), 3473-89.

- Bliss, D. (2009). Lean in healthcare-wow. Frontiers of Health Services Management, 26(1), 39-42

- Brandao, L. (2009). Trends and approaches in lean healthcare. Leadership in Health Services, 22(2), 121-139

- Breuer, D. J. (2013). Lean implementation in healthcare - the challenged transformation. IIE Annual Conference.Proceedings, 1265-1274.

- $\quad$ Burgess, N., y Radnor, Z. (2013). Evaluating lean in healthcare. International Journal of Health Care Quality Assurance, 26(3), 220-35

- Cachon, G. y Terwiesch, C. (2009). Matching Supply and Demand, International Edition. Singapore: McGraw-Hill.

- Cristina, M. y Crespo, D. (2013). Strategic outsourcing: A lean tool of healthcare supply chain management. Strategic Outsourcing: An International Journal, 6(2), 138-166.

- Culcuoglu, M.; Wang, S.; Nice, L.; Caslow, A.; Stedman, P. y Foster, S. (2011). Continuous perioperative process improvement using lean principles. IIE Annual Conference Proceedings, 1-8.

- Dinas, J.; Franco, P. y Rivera, L. (2009). Aplicación de herramientas de pensamiento sistémico para el aprendizaje de Lean Manufacturing. Universidad ICESI, 7(14), 10944.

- $\quad$ Fortuny, J.; Cuatrecasas, L.; Cuatrecasas, O. y Olivella, J. (2008). Metodología de implantación de la gestión lean en plantas industriales. Universia Business Review, 28-41.

- $\quad$ Found, P. y Harrison, R. (2012). Understanding the lean voice of the customer. International Journal of Lean Six Sigma, 3(3), 251-267.

- Gifford, C. (2008). A lean, mean, continuous improvement machine. INTECH, 55(2), 64.

- Waring, J. y Bishop, S. (2010). Lean healthcare: Rhetoric, ritual and resistance. Social Science \& Medicine, 71(7), 1332-1340.

- Kollberg, B., Dahlgaard, J. y Per-Olaf Brehmer. (2007). Measuring lean initiatives in health care services: Issues and findings. International Journal of Productivity and Performance Management, 56(1), 7-24.

- Lasa, I.; Laburu, C. y Vila, R.C., (2008). An evaluation of the value stream mapping tool. Business Process Management, 14 (1), 39-52.

- Lewis, J.(2012). Whylean? CabinetMaker+FDM, 26(3), 18-18,20.

- Laganga, L.R. (2011). Lean service operations: Reflections and new directions for capacity expansion in outpatient clinics, Journal of Operations Management, 29 (5) 422-433.

- Malmbrandt, M. y Åhlström, P. (2013). An instrument for assessing lean service adoption. International Journal of Operations \& Production Management, 33(9), 1131-1165

- Mannon, M. (2014). Lean healthcare and quality management: The experience of ThedaCare. The Quality Management Journal, 21(1), 7-10.

- $\quad$ Manos, A.; Sattler, M. y Alukal, G. (2006). Make healthcare lean. Quality Progress, 39(7), 24-30.

- Martínez, P., Martínez, J.; Nuño, P. y Cavazos, J. (2015). Mejora en el tiempo de atención al paciente en una unidad de urgencias mediante la aplicación de manufactura esbelta. Información Tecnológica, 26(6), 187-198. https://dx.doi. org/10.4067/S0718-07642015000600019

- Martínez, P.; Martínez, J.; Nuño, P. y Cavazos, J. (2014). Process improvement in Emergency Units. Two Analysis cases. IIE Annual Conference.Proceedings, 1-9.

- Mazzocato, P., Holden, R. J., Brommels, M., Aronsson, H., Bäckman, U., Elg, M., y Thor, J. (2012). How does lean work in emergency care? A case study of a lean-inspired intervention at the astrid lindgren children's hospital, stockholm, sweden. BMC Health Services Research, 1228.

- Moreno, M. A y Mendoza, A. (2012). Hospital excellence operation model: An approach to lean healthcare in mexican hospitals. IIE Annual Conference Proceedings, 1-10.

- $\quad$ Papadopoulos, T.; Radnor, Z. y Merali, Y. (2011). The role of actor associations in understanding the implementation of lean thinking in healthcare. International Journal of Operations \& Production Management, 31(2), 167-191

- Pestana, A. L, Dos Santos, J.; Erdmann, R.; Da Silva, E. y Erdmann, A. (2013). Lean thinking and brain-dead patient assistance in the organ donation process. Revista da Escola de Enfermagem da USP, 47(1), 258-264 
- $\quad$ Piggott, Z.; Weldon, E.; Strome, T. y Chochinov, A. (2011). ED administration: Application of lean principles to improve early cardiac care in the emergency department. CJEM: Journal of the Canadian Association of Emergency Physicians, 13(5), 325-32.

- $\quad$ Ponanake, P.; Limnararat, S., Pithuncharurnlap, M., y Sangmanee, W. (2014) Creating hospitals' core competencies with lean healthcare strategies entering the ASEAN economic community. Journal of Economics and Behavioral Studies, 6(9), 700-708.

- $\quad$ Pondhe, R.; Asare, S.; Badar, M.; Zhou, M. y Leach, R. (2006). Applying lean techniques to improve an emergency department. IIE Annual Conference.Proceedings, 1-6.

- Reijula, J. y Tommelein, I. (2012). Lean hospitals: A new challenge for facility designers. Intelligent Buildings International, 4(2), 126-143.

- Sobek, D. y Lang, M. (2010). Lean healthcare: Current state and future directions. IIE Annual Conference Proceedings, 1-6.
- $\quad$ Toussaint, J. y Berry, L. (2013). The promise of lean in health care. Mayo Clinic Proceedings, 88(1), 74-82.

- $\quad$ Varkey, P. y Kollengode, A. (2011). framework for healthcare quality improvement in india: The time is here and now! Journal of Postgraduate Medicine, 57(3), 237-41.

- $\quad$ Vinodh, S.; Arvind, K. y Somanaathan, M. (2010). Application of value stream mapping in an indian camshaft manufacturing organisation. Journal of Manufacturing Technology Management, 21(7), 888-900.

- Weinstock, D. (2008). Lean healthcare. The Journal of Medical Practice Management, 23(6), 339-41.

- Wood, N. (2004). Customer value: Applying the first principle of lean. Management Services, 48(3), 14-17.

- Zarbo, R. (2012). Creating and sustaining a lean culture of continuous process improvement. American Journal of Clinical Pathology, 138(3), 321-326 